\title{
Characterizing variation in mycorrhiza effect among diverse plant varieties
}

\author{
Ruairidh J. H. Sawers • Mesfin N. Gebreselassie • \\ David P. Janos $\cdot$ Uta Paszkowski
}

Received: 11 May 2009/Accepted: 21 November 2009/Published online: 15 December 2009

(C) Springer-Verlag 2009

\begin{abstract}
Exploitation of arbuscular mycorrhizal fungi may be an important approach for development of reducedinput agriculture. We discuss the use of linear models to analyze variation in mycorrhiza response among diverse plant varieties in order to assess the value of mycorrhizas. Our approach allows elimination of variation linked to differences in plant performance in the absence of mycorrhizas and the selection of plant lines that might harbor genetic variation of use to improve the mycorrhizal symbiosis in agriculture. We illustrate our approach by applying it to previously published and to novel data. We suggest that in dealing with a relative trait such as mycorrhiza effect, the choice of measure used to quantify the trait greatly affects interpretation. In the plant populations under consideration, we find evidence for a greater potential to increase mycorrhiza benefit than previously suggested.
\end{abstract}

\section{List of symbols}

NC Performance of non-mycorrhizal plants

$M \quad$ Performance of mycorrhizal plants

$R \quad$ Absolute responsiveness

Communicated by E. Guiderdoni.

Electronic supplementary material The online version of this article (doi:10.1007/s00122-009-1231-y) contains supplementary material, which is available to authorized users.

R. J. H. Sawers $(\bowtie) \cdot$ M. N. Gebreselassie · U. Paszkowski Department of Plant Molecular Biology, University of Lausanne, Biophore Building, 1015 Lausanne, Switzerland e-mail: ruairidh.sawers@unil.ch

D. P. Janos

Department of Biology, University of Miami, P.O. Box 249118, Coral Gables, FL 33124, USA
$R^{\prime} \quad$ Relative responsiveness, relative to NC

$\mu(X) \quad$ Mean of $X$

$\sigma^{2}(X) \quad$ Variance of $X$

$\sigma(X, Y)$ Covariance of $X$ and $Y$

$i \quad$ Intercept of regression line

a Slope of regression line

$\delta \quad$ Deviation from regression line

$a_{M} \quad$ Slope of the regression line of $M$ against NC

$a_{R} \quad$ Slope of the regression line of $R$ against NC

$i_{M} \quad$ Intercept of the regression line of $M$ against NC

$i_{R} \quad$ Intercept of the regression line of $R$ against NC

$r^{2} \quad$ Coefficient of determination

$r_{M}^{2} \quad$ Coefficient of determination of $M$ against NC

$r_{R}^{2} \quad$ Coefficient of determination of $R$ against NC

$m \quad$ Asymptote of a logistic dose-response curve

$n \quad y$-axis intercept of a logistic dose-response curve

$f \quad$ Position of the inflection point of a logistic dose-response curve

d Mycorrhiza dependence (see Janos 2007) for $15 \%$ of a logistic dose-response curve asymptote

\section{Introduction}

Global agricultural phosphate use has risen to almost 40 million tons per year, the majority being used for cereal crops (http://www.fao.org). The bulk of this demand is satisfied by the mining and processing of rock phosphate, a limited-resource that will cease to be viable before the end of this century (Khasawneh et al. 1986). The efficiency of uptake of added phosphate can be as low as $20 \%$ (Zhu et al. 2003) and, if not immobilized, much may pass directly to 
water courses with detrimental environmental consequences (Delgado and Scalenghe 2008). It is clear that current levels of agricultural phosphate use are neither desirable nor sustainable. Today's crop yields, however, depend upon these additions, and commercial cereal varieties have been selected precisely because of their ability to thrive in high-input agriculture (Evenson and Gollin 2003). It is now widely acknowledged that one of the key challenges facing the agricultural and plant science community is the maintenance of yields, while reducing dependence on fertilizers (http://www.fao.org). Among the many avenues of research that may contribute to the resolution of this problem, the exploitation of arbuscular mycorrhizal fungi (AMF) has been proposed as a way to improve the efficiency of phosphate use in agricultural systems (Sawers et al. 2008).

The majority of terrestrial plants live in symbiotic association with AMF that grow both within, and surrounding, their roots (Smith and Read 2007). The fungi rely upon plant carbon for growth and completion of their life-cycle. In return, the host plant receives mineral nutrients acquired by a network of extra-radical hyphae that effectively extend its root system. Where examined, crop species have retained the capacity of their wild-growing relatives to form associations with AMF and to benefit from them, especially when nutrients are limiting (e.g., Hetrick et al. 1992; Kaeppler et al. 2000; Koide et al. 1988). Under high-nutrient conditions, however, the benefit of the symbiosis usually is diminished, and growth depression by mycorrhizas has been observed in several species (Grace et al. 2009; Li et al. 2008; Smith et al. 2009). The challenge for plant breeders who wish to improve utilization of AMF in agriculture is to devise a sound conceptual framework for comparison of plant varieties and growing conditions, and for selection of improved crop germplasm (Sawers et al. 2008).

Selection simply to maximize the difference in yield between individuals grown in the presence or absence of AMF, often referred to as the response to AMF, may not identify agriculturally useful variation. Plants can show a large response to mycorrhizal fungi because of their inability to grow without them (Janos 2007). For example, maize mutants with disrupted root morphology that grow exceptionally poorly when nutrients are limiting can approach wild-type performance when colonized by AMF, thus are highly responsive (Paszkowski and Boller 2002). Furthermore, poor performance of cereal varieties under limiting nutrient conditions in the absence of mycorrhizas often is correlated with the greatest response to AMF (e.g., Hetrick et al. 1992; Kaeppler et al. 2000; Koide et al. 1988; Zhu et al. 2001). To describe this aspect of mycorrhiza response, Janos (2007) has defined the property plant "dependence", quantified relatively as the level of nutrient availability required by plants to achieve an arbitrary minimum level of performance in the absence of AMF. A large response to AMF that is attributable to high dependence likely is of little interest to plant breeders because at best it may be irrelevant to maximizing yield and at worst it may conflict with that objective. Nonetheless, it is important to take variation in the performance of non-colonized plants into account, especially when the plant varieties under consideration are diverse and their performance varies widely. Moreover, when the lines under consideration are inbred lines, historical varieties, or landraces, the overall level of performance will typically be inferior to that of an elite cultivated variety even for those lines that harbor potentially beneficial variation. To address this, Sawers et al. (2008) suggested partitioning variation in response to AMF into dependence-based and non-dependence-based components. It is the second of these sources of variation, i.e., differences in response that are not linked to variation in the performance of non-colonized plants, that is of greatest interest to plant breeders and geneticists working to optimize AMF for agricultural use.

The difficulties of comparing responses to colonization by AMF illustrate the general problem of analyzing variation in a trait that is based on a relative measure or index (Sawers 2009). While it can be attractive to assess plant qualities on the basis of relative performance between two growth conditions (e.g., with or without fertilizer, in the presence or absence of pathogens, under low or high salinity) there are a number of difficulties in considering a relative response in the same way as an absolute trait such as height or yield. The extent of the covariance in performance between the two environmental or treatment conditions cannot be estimated a priori (Falconer 1990). Where there is genetic potential for a degree of independence in variation, outlying levels of response can result from variation in performance under either condition. Typically, however, there is more interest in one growth condition than the other, with the aim not to identify the variety that performs exceptionally in the absence of fertilizer, when not diseased, under low salinity, or when not colonized by AMF. Therefore, it is useful to treat the two performance measures that constitute a response as distinct traits, and to make no initial assumption concerning their degree of co-variation in any given population.

Here, we present a method to eliminate the contribution to variation in plant response to AMF of differences in performance among non-colonized plants. We propose that the residual, non-dependence-based, variation can be employed to identify plant genetic variation useful in enhancing AMF promotion of crop yields. In addition, we compare the expectations of our method with the often-used ranking of varieties based on response measures. By analyzing both previously published and novel data, we re-examine the importance of AMF to modern crop varieties, and we assess co-variation of the performance of mycorrhizal and noncolonized plants. In line with prevailing research interest, we predominantly consider the impact of mycorrhizas on plant 
nutrient uptake, and we emphasize the role of the symbiosis in plant phosphorus acquisition. Nevertheless, our method is applicable to other phenotypic traits. Although we will draw the majority of our examples from the study of major cereal crops, our comments also apply to other facultatively mycotrophic (see Janos 2007) plant populations.

\section{Materials and methods}

Plant growth and performance measurement

Two maize inbred lines, Oh43 and Pa36, were grown with or without inoculation with AMF over a range of phosphate $\left(\mathrm{P}_{\mathrm{i}}\right)$ availability. Factorial treatment combinations comprised six $\mathrm{P}_{\mathrm{i}}$ levels $(0,50,100,200,400,800 \mu \mathrm{M})$, two mycorrhizal treatments (non-mycorrhizal and mycorrhizal), and the two maize genotypes. These 24 treatment combinations were grown in a randomized complete block design with 5 replications for a total of 120 experimental units. Seeds were germinated in quartz sand for 2 weeks. Seedlings were then transplanted to $1,000-\mathrm{ml}$ plastic pots filled with $750 \mathrm{ml}$ of autoclaved loam: sand mixture (1:10). Plants were grown in greenhouse conditions maintained at $50 \%$ humidity and $28^{\circ} \mathrm{C}$ day/night temperature with a $12-\mathrm{h}$ light period, including supplementary light when required. For mycorrhizal treatments, a mycorrhizal inoculum consisting of dry soil containing Glomus mosseae spores, hyphae, and infected roots generated from plants (Tagetes harmony) grown in this same soil was mixed with the autoclaved soil mixture (Paszkowski and Boller 2002). Plants were fertilized three times per week with $100 \mathrm{ml}$ of modified $1 \times$ standard Hoagland solution (Hoagland and Broyer 1936) containing variants of $\mathrm{KH}_{2} \mathrm{PO}_{4}$ concentrations according to the $\mathrm{P}_{\mathrm{i}}$ dose. The concentration of potassium was maintained constant by addition of $\mathrm{KCl}$ as required. Plants were harvested $\approx 8$ weeks after emergence and shoot dry weight was determined for each individual. Root samples were randomly collected, and colonization by AMF was quantified with stained roots by assessing the presence or absence of fungal structures (intra-radical hyphae, vesicles, and arbuscules) at 100 intersect-points per root as described by Gutjahr et al. (2008).

\section{Results}

Methodology

\section{Context and assumptions}

To discuss intra-specific variation in the performance of mycorrhizal plants, we consider the analysis of a relatively large number of diverse, inbred varieties that provide a good sample of the broader population from which they were derived. We assume the availability of measures of performance of mycorrhizal $(M)$ and noncolonized plants (NC) that accurately reflect the true genetic value of each variety and allow meaningful comparison among varieties. Throughout our discussion, we will not consider either environmental or error variation. The measures $M$ and NC, however, do not need to relate to a single trait under a given environmental condition; they might represent parameters describing doseresponse behavior, performance integrated over a number environments, or arrays containing multiple performance scores. We assume that the conditions are such that there is a performance increase between mycorrhizal and noncolonized plants, and refer to such a difference as the mycorrhiza effect, a term we use in a purposefully broad and qualitative sense. In contrast, we will strictly define and compare a number of measures aimed at quantifying the mycorrhiza effect.

\section{Partitioning variation in mycorrhiza effect}

To partition variation in mycorrhiza effect, we present a linear model that describes population-level variation in the relationship between mycorrhiza effect and the performance of non-colonized plants. We begin by examining the use of absolute responsiveness as a measure of mycorrhiza effect. Following Janos (2007), absolute responsiveness $(R)$ is defined as the difference between the performance of mycorrhizal and non-colonized plants (Eq. 1).

$R=M-\mathrm{NC}$.

In a population, variation in $R$ can be partitioned into variation correlated with $\mathrm{NC}$ and other variation. Using a linear model, we can describe $R$ as a function of NC and deviations,

$R=i+a \mathrm{NC}+\delta$

where $i$ is the axis intercept, $a$ is the slope, and $\delta$ are the deviations. Variation in NC between plant varieties will result both from differences in general growth requirements and from variation in plant components required specifically for asymbiotic growth. The former are expected also to affect the growth of mycorrhizal plants and to contribute to variation in $M$. The balance of the variation in $M$ results from differences in components specific to mycorrhizal growth. It is those components that we wish to identify by using a genetic approach, and it is high-performing variation in those components that forms the logical target of breeding efforts to improve plant performance with mycorrhizas. 
Substitution of Eq. 1 into Eq. 2 shows that regression of $R$ on $\mathrm{NC}$ is equivalent to the direct regression of $M$ on $\mathrm{NC}$ (Eq. 3)

$M-\mathrm{NC}=i+a \mathrm{NC}+\delta$

$M=i+(a+1) \mathrm{NC}+\delta$.

Notably, the intercept and deviations are the same in the two regression equations and the slope of the regression of $R$ on NC is simply the slope of $M$ on NC minus 1 . We emphasize that the key quantity is $\sigma(M, \mathrm{NC})$, and that calculation of $R$ reveals nothing new about this relationship.

Variation in $M$ can be partitioned using standard methods (Lynch and Walsh 1998) into that described by the regression on NC and deviations (Eq. 4)

$$
\begin{aligned}
\sigma^{2}(M) & =\sigma^{2}(\text { regression }+\delta) \\
& =\sigma^{2}(\text { regression })+\sigma(\text { regression }, \delta)+\sigma^{2}(\delta) \\
& =\sigma^{2}(\text { regression })+\sigma^{2}(\delta) .
\end{aligned}
$$

The partition shown in Eq. 4 is similar to the distinction previously made by Sawers et al. (2008) between dependence-based and non-dependence-based variation. Here, we propose the more-accurate terms common and specific to describe the regression and deviation variance components, respectively; common variation, $\sigma^{2}$ (regression), affects the performance of both noncolonized and mycorrhizal plants; specific variation, $\sigma^{2}(\delta)$, represents outlying variation acting specifically on noncolonized or mycorrhizal plant performance. It is important to emphasize that the regression variance includes the covariance $\sigma(M, \mathrm{NC})$ and thereby includes any mycorrhizal effect shared by all varieties, including the effects of so-called "mycorrhizal-specific genes" (e.g., Güimil et al. 2005; Gutjahr et al. 2008); i.e., given a non-zero value for the intercept, an absence of deviation variation would not suggest an absence of mycorrhiza effect but rather an absence of specific variation. Where present, deviations represent outlying variation acting specifically on noncolonized or mycorrhizal plant performance alone. A positive deviation associated with a given line represents a level of performance of mycorrhizal plants above that expected from non-colonized performance in a given population. Such positive deviations are candidates for the beneficial non-dependence-based variation described by Sawers et al. (2008).

The importance of common variation in determining differences in $M$ in a given population is indicated by the coefficient of determination $\left(r^{2}\right)$ of the regression (Eq. 5)

$r_{M}^{2}=\frac{\sigma(M, \mathrm{NC})^{2}}{\sigma^{2}(M) \times \sigma^{2}(\mathrm{NC})}$.

In the simple case of a standardized data set where $\sigma^{2}(M)=\sigma^{2}(\mathrm{NC})=1$,
$r_{M}^{2}=\sigma(M, \mathrm{NC})^{2}$

For a given population, the magnitude of the specific variation $\left(1-r_{M}^{2}\right)$ is an important consideration in determining the suitability for a mycorrhiza genetic or breeding analysis. Note that although the regressions of $M$ and $R$ on NC have equal intercepts and the same deviations, the value of $r^{2}$ for the latter regression (Eq. 7) differs from that of the former

$r_{R}^{2}=\frac{\sigma(R, \mathrm{NC})^{2}}{\sigma^{2}(R) \times \sigma^{2}(\mathrm{NC})}$.

Substituting Eq. 1 for $R$, Eq. 7 can be expressed in terms of $M$ and NC (Eq. 8)

$r_{R}^{2}=\frac{\sigma(M-\mathrm{NC}, \mathrm{NC})^{2}}{\sigma^{2}(M-\mathrm{NC}) \times \sigma^{2}(\mathrm{NC})}$.

In the simple case described above (Eq. 6) this is equivalent to,

$$
\begin{aligned}
r_{R}^{2} & =\frac{\left[\sigma(M, \mathrm{NC})-\sigma^{2}(\mathrm{NC})\right]^{2}}{\left[\sigma^{2}(M)+\sigma^{2}(\mathrm{NC})-2 \sigma(M, \mathrm{NC})\right] \times \sigma^{2}(\mathrm{NC})} \\
& =\frac{\left[r_{M}-1\right]^{2}}{\left[1+1-2 r_{M}\right] \times 1} \\
& =\frac{1-r_{M}}{2} .
\end{aligned}
$$

Therefore, the relative contribution of common variation will be different depending on whether $M$ or $R$ is taken as the measure of mycorrhiza effect. Notably, in this case, as $r_{M}^{2}$ becomes small, i.e., in the absence of co-variation of $M$ and NC, the value of $r_{R}^{2}$ will tend to 0.5 .

\section{Other measures of mycorrhiza effect}

To compare mycorrhiza effect, researchers have used a variety of measures in addition to the absolute difference between $M$ and NC (see Table 1 in Janos 2007). A widely used approach has been to weight the absolute difference relative to the performance of mycorrhizal or non-colonized plants. Such relative measures can be generalized by consideration of the ratios $M / \mathrm{NC}$ and $\mathrm{NC} / M$. We note that in addition to being used directly, these performance ratios are equivalent to commonly quoted fold-change or percentage-change measures (discussed in Janos 2007). For example, the fold-change relative to $\mathrm{NC}$, that we designate $R^{\prime}$, can be calculated as:

$R^{\prime}=\frac{M-\mathrm{NC}}{\mathrm{NC}}$.

Equation 10 can be re-arranged as:

$R^{\prime}=\frac{M}{\mathrm{NC}}-1$. 
The ratio of $M$ over NC is equivalent to the differences of logarithmic transformations of NC and $M$ and the regression of $\log \left(R^{\prime}\right)$ against $\log \left(\mathrm{NC}^{\prime}\right)$ can be expressed as previously described for that of $R$ against NC. If the relationship between $\log \left(R^{\prime}\right)$ and $\log \left(\mathrm{NC}^{\prime}\right)$ is linear, however, the relationship between $R^{\prime}$ and $\mathrm{NC}$ will follow a power model. Consequently, in such cases, analyses that consider the relationship between $R^{\prime}$ and $\mathrm{NC}$ as linear will be biased, especially when considering varieties that exhibit extreme levels of NC.

\section{Application to experimental data}

To examine the ideas presented above using experimental data, we have reassessed two of the most significant studies of crop mycorrhiza effect: a collection of works addressing diversity in AMF interactions in wheat (Hetrick et al. 1992, 1995, 1996) and maize (Kaeppler et al. 2000). For our purposes, we take treatment means from these studies to be good estimates of $M$ and NC. Although there are additional sources of variation present in these experimental data, our analyses illustrate our foregoing presentation. We further investigate conclusions arising from our analysis of the published maize data with our own experiment.

\section{Mycorrhiza effect in modern and landrace wheat sub-populations}

Hetrick and co-workers performed a comparative analysis among cereal crop varieties and sub-populations (Hetrick et al. 1992, 1995, 1996). Although the authors considered a number of small-grains in their work, their most extensive dataset involved Triticum aestivum, for which they collected performance data from mycorrhizal and non-colonized plants of 13 modern varieties and 7 landraces (Hetrick et al. 1992). On the basis of their analysis they drew a number of important conclusions, the most relevant to the present discussion being that mycorrhiza effect is lower in modern crop varieties than in related landrace populations (Hetrick et al. 1992). The authors went on to hypothesize that the breeding process, conducted under high-input agricultural conditions, "could have reduced the frequency of genes that foster the mycorrhizal associations." In later work, they conclude that lines for which mycorrhiza effect is small are "lacking responsiveness genes" (Hetrick et al. 1996). This thought-provoking hypothesis remains central to the question of the agricultural utility of AMF, and it has been investigated further in wheat (Zhu et al. 2001) and other cereals (Koide et al. 1988; Wright et al. 2005). In their published analysis, Hetrick and co-workers use the relative responsiveness measure $R^{\prime}$ expressed as a percentage. In their interpretation, the authors make an explicit link between differences in $R^{\prime}$ among varieties and the action of genes that we would link to specific variation. Following the above discussion, however, we hypothesize that a proportion of the variation in $R^{\prime}$ might be linked to differences in $\mathrm{NC}$ performance, i.e., what we have called common variation.

To investigate co-variation of $M$ and $\mathrm{NC}$ in wheat, and to address the hypothesis that modern and landrace varieties differ with respect to mycorrhiza effect, we analyzed the T. aestivum data published by Hetrick et al. (1992). We performed a linear regression of $M$ against $\mathrm{NC}$ (measured as shoot dry weight) for the data set as a whole. The combined data shows a strong correlation between $M$ and NC $\left(r^{2}=0.74\right)$, a positive intercept $(i=0.82)$, and a positive slope $(a=1.1)$. Importantly, both landrace and modern varieties fell along the same regression line for $M$ against NC (Fig. 1). While there is evidence of only limited specific variation in this population $\left(1-r^{2}=0.26\right)$, we analyzed residuals to identify outlying lines. The varieties with the largest positive (cultivar Avalon) and negative (cultivar Newton) residuals both are modern lines, consistent with equivalence between the sub-populations (Table S1). Where the sub-populations do differ clearly is in the range of performance of non-colonized plants $(t$ test of mean NC between modern and landrace populations: $P<0.01)$. The landraces are over-represented among the most poorly performing plants, and it is this distribution, given the negative correlation between $R^{\prime}$ and NC, which principally results in a higher mean $R^{\prime}$ for the landraces than for the modern varieties. We conclude that the correlation between the performance of non-colonized and mycorrhizal plants is similar for both modern and landrace populations. There is no evidence to suggest that modern varieties have lost the ability to respond to mycorrhizal colonization, only that in this collection under the conditions of the experiment they show, on average, better performance when non-colonized than do the landraces.

\section{Identification of suitable maize parents to isolate genes involved in AM symbioses}

In their study, Kaeppler and co-workers quantified mycorrhiza effect in 28 mid-west US-adapted maize inbred lines (Kaeppler et al. 2000). The aims of their work were to directly identify potentially useful variation in mycorrhiza effect and to identify outlying parents for further genetic analysis. Plant performance was measured as the shoot drymass of 6-week-old, greenhouse-grown plants. Following the analyses of Hetrick et al., mycorrhiza effect was calculated as $R^{\prime}$ (Eq. 13).

Kaeppler et al. (2000) found that under low phosphorus availability all lines exhibited a positive growth response to mycorrhizal colonization. Values of $R^{\prime}$ (as a percentage) 


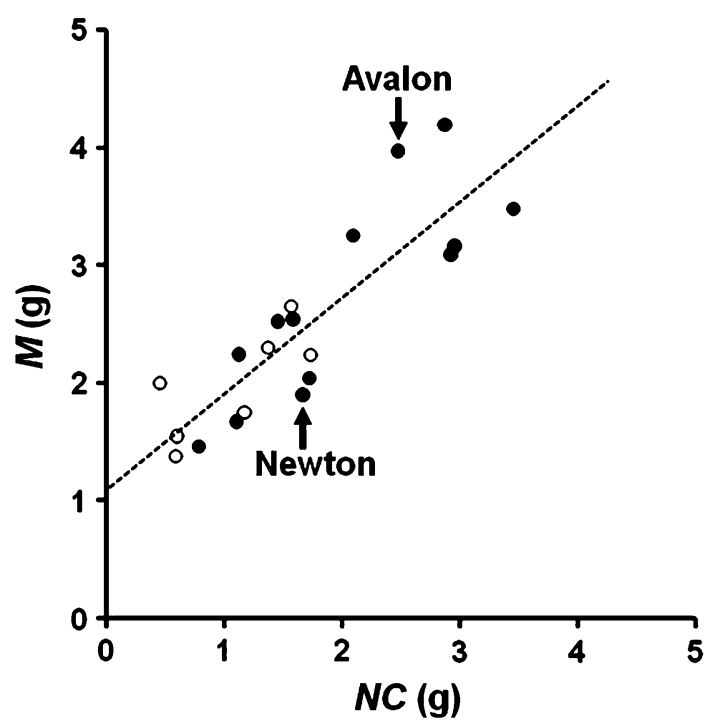

Fig. 1 Regression of shoot dry-mass of mycorrhizal plants $(M)$ against that of non-colonized plants $(N C)$ for 13 modern wheat varieties and 7 landraces (Hetrick et al. 1992). Modern wheat varieties are shown as filled circles. Landrace wheat varieties are shown as open circles. Lines discussed in the text are labeled

ranged from a maximum of $800 \%$ (line B73) to a minimum of $106 \%$ (line Mo17). Consequently, the authors selected a population of $\mathrm{B} 73 \times \mathrm{Mo17}$ derived recombinant inbred lines (RILs) to screen for detection of quantitative trait loci (QTL) associated with mycorrhiza effect. The loci subsequently identified, however, were found predominantly to be associated with variation in the performance of noncolonized plants. From their analysis as a whole, the authors concluded that "differences in mycorrhizal responsiveness measured among inbred lines are determined primarily by variation among genotypes for growth at low phosphate in the absence of mycorrhizae and much less by variation in genotypes for their ability to respond to mycorrhizae"; i.e., in this population, differences in $M$ are dominated by variation in common components. As stated previously, we propose that it is specific variation that is important from the perspective of crop improvement. Therefore, we sought to use partitioning of variance in mycorrhiza effect to statistically eliminate the observed NC differences and to identify lines with an outlying "ability to respond".

On the basis of our methodology section, we predicted that the choice of measure of mycorrhiza effect would greatly influence interpretation of the Kaeppler et al. (2000) data, especially when an expectation was based on a presumed linear correlation between $R^{\prime}$ and NC. We calculated three different measures, $M, R$, and $R^{\prime}$, from the low-phosphate performance data presented in Table 1 of Kaeppler et al. (2000), and we performed regression analyses of the three measures on NC (Fig. 2; Table 1).
Regression of $M$ on NC reveals only a weak relationship between the performance of non-colonized and mycorrhizal plants (Fig. 2a); differences in NC have little effect on the expected value of $M(a=0.54)$, and the level of covariance between $M$ and NC is low relative to the level of variation within the two treatment groups $\left(r^{2}=0.14\right)$. This result clearly is not consistent with the published conclusion that there is little specific variation in this population. For this dataset, an analysis based on $R$ gives a similar overall picture of the population; the position of the intercept remains unchanged, the slope is transformed by -1 and the value of $r^{2}$ is comparable $\left(r^{2}=0.10\right)$ (Fig. 2b). The regression of $R^{\prime}$ against NC can be well modeled using a power relationship $\left(r^{2}=0.77\right)$ (Fig. 2c). The linear fit, however, although strong $\left(r^{2}=0.64\right)$ is clearly biased, especially at the high and low extremes of NC. There is no mathematical inconsistency between the coefficients of determination obtained with the different measures of mycorrhiza effect. There is, however, inconsistency in the biological interpretation. From a linear correlation between $R^{\prime}$ and NC, Kaeppler et al. (2000) stated that the majority of the variation in $R^{\prime}$ is explained by variation in NC. Although that is correct, we propose that the regression based on $M$, and the associated lower coefficient of determination, may best reflect co-variation of the performance of colonized and non-colonized plants and especially the true extent of specific variation in this population.

The steep regression slope and strong correlation between $R^{\prime}$ and NC mean that the ranking of individuals on the basis of $R^{\prime}$ will emphasize differences in the performance of non-colonized plants. Consequently, the lines selected as outliers by Kaeppler et al. (2000) (B73 and Mo17) were both the most extreme for $R^{\prime}$ and for NC, which is consistent with the subsequent finding of the QTL analysis. We consider these two lines to exhibit a dependence-based difference in responsiveness, the magnitude of which is exaggerated by the use of the measure $R^{\prime}$. Following regression of $M$ or $R$ against $\mathrm{NC}, \mathrm{B} 73$ and Mo17 fall close to the regression line, suggesting that the difference in $M$ between the two lines is well explained by the level of $\mathrm{NC}$ growth, i.e., there is little evidence that the two inbred lines differ with regard to specific components or that they are exceptional in that regard (Fig. 2a, b). Similarly, B73 and Mo17 fall along the regression line of $R^{\prime}$ against NC when a power model is used (Fig. 2c). To identify alternative candidate lines outlying for mycorrhiza effect, we examined the deviations $(\delta)$ from the regression analyses of $M$ on NC (Fig. 3; Table S2). The line Pa36 was identified when mycorrhizal as performing worse than expected from its $\mathrm{NC}$ value. In addition, a small group of lines, W37A, Oh43, and W59M, have levels of $M$ exceeding the population expectation (Fig. 3). Although the lines $\mathrm{Oh} 43$ and $\mathrm{Pa} 36$ also are identified by using $R^{\prime}$ (not 

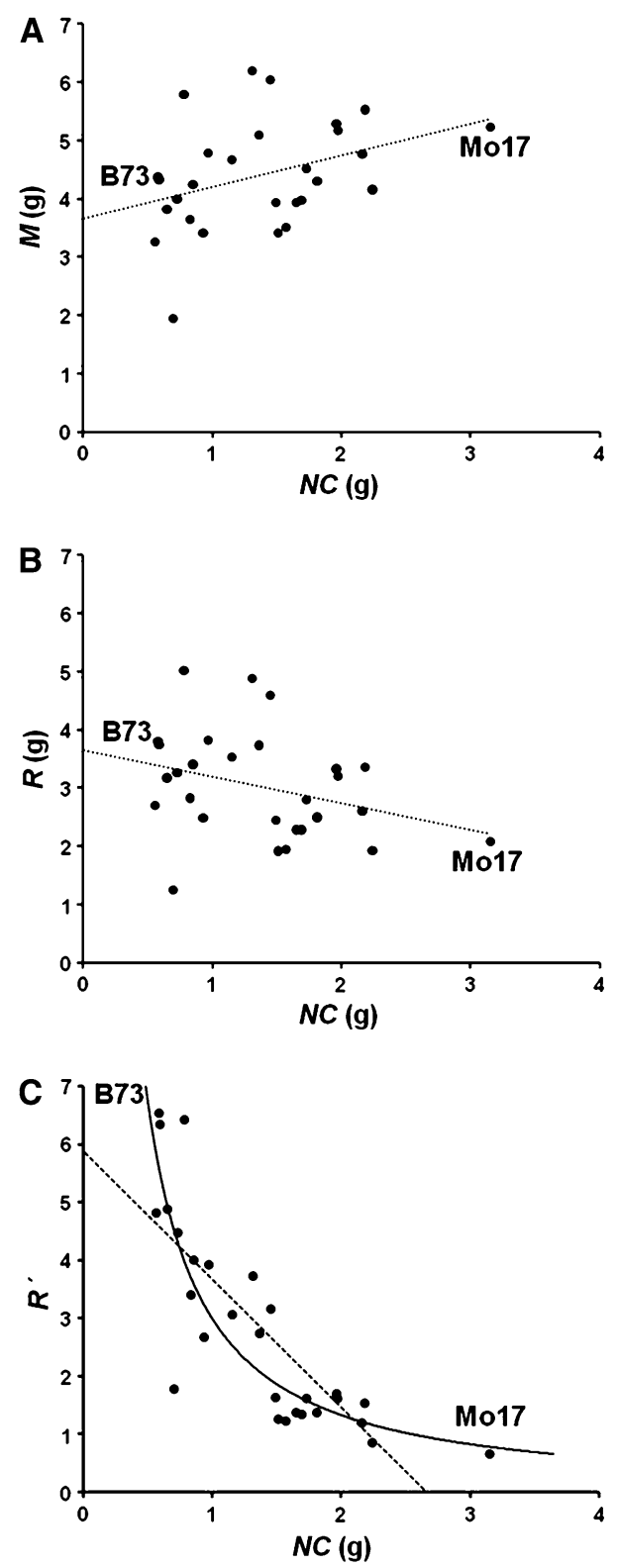

Fig. 2 Regression of measures of mycorrhiza effect against the shoot dry-mass of non-colonized plants $(N C)$ for 28 maize inbred lines grown under low-phosphate (Kaeppler et al. 2000). a Shoot dry-mass of mycorrhizal plants $(M)$ against NC. b Absolute responsiveness $(R)$ against NC. c Relative responsiveness $\left(R^{\prime}\right)$ against NC. Data-points corresponding to the maize lines B73 and Mo17 are labeled. Linear regression $(\mathbf{a}, \mathbf{b}$ and $\mathbf{c})$ and power regression (c) trend-lines are shown

shown), the lines W37A and W59M are not exceptional in the latter regression, a discrepancy that is consistent with the emphasis placed on $\mathrm{NC}$ by the use of $R^{\prime}$. We propose that the measures $M$ or $R$ give a truer reflection of mycorrhiza effect than does $R^{\prime}$, and that the lines Pa36, W37A, Oh43, and W59M potentially harbor variation in specific components which makes them good candidates for further study.
Table 1 Measures of mycorrhiza effect and regression analysis of the maize low-phosphate performance data of Kaeppler et al. (2000)

\begin{tabular}{llllllll}
\hline Measure $(x)$ & $\mu(x)$ & $\sigma^{2}(x)$ & $\sigma(x, \mathrm{NC})$ & $a$ & $i$ & $r^{2}$ & $1-r^{2}$ \\
\hline NC & 1.40 & 0.40 & N/A & N/A & N/A & N/A & N/A \\
$M$ & 4.40 & 0.85 & 0.22 & 0.54 & 3.65 & 0.14 & 0.86 \\
$M-\mathrm{NC}$ & 3.00 & 0.81 & -0.18 & -0.46 & 3.65 & 0.10 & 0.90 \\
$\frac{M-N C}{\mathrm{NC}} \times 100$ & 283 & 30,260 & -88 & -221 & 588 & 0.64 & 0.36 \\
\hline
\end{tabular}

$M$ shoot dry-mass of mycorrhizal plants (g), $N C$ shoot dry-mass of non-colonized plants $(\mathrm{g})$

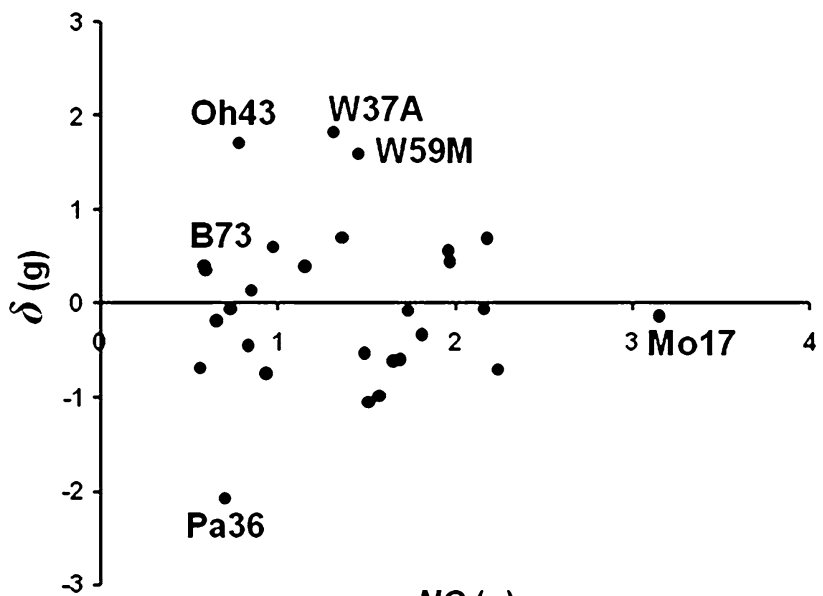

$N C(g)$

Fig. 3 Residual plot for regression of the shoot dry-mass of mycorrhizal plants $(M)$ against the dry mass of non-colonized plants $(N C)$ for 28 maize inbred lines grown under low-phosphate (Kaeppler et al. 2000). Lines discussed in the text are labeled

\section{Phosphate dose-response behavior of the maize lines $\mathrm{Oh} 43$ and Pa36}

Our identification of outlying maize lines Oh43 and Pa36 from the Kaeppler et al. (2000) data set was based on performance measures obtained under a single, low-phosphate environmental condition. As was recognized by Hetrick et al. (1996), however, the level of mycorrhiza effect will change depending upon the environmental conditions. Not only can mycorrhiza effect change among environments, but the way it changes also can differ among plant genotypes. Formally, such differences are described as genotype $\mathrm{X}$ phenotype interactions, and they can result in re-ranking of varieties in different environments (Falconer 1990). Therefore, the difference observed between the lines $\mathrm{Oh} 43$ and $\mathrm{Pa} 36$ could result because the low-phosphate condition fell at different positions along the dose-response relationships of the lines (Janos 2007). Although a full genetic analysis of the differences between Oh43 and Pa36 will require the generation of novel mapping populations, it is possible to investigate $M$ and $\mathrm{NC}$ phosphate dose-response relationships for these lines, as 
described by Janos (2007). In particular, such an investigation addresses the major concern arising from the single environment analysis, i.e., that the response of the lowperforming inbred line is underestimated and that under a diminished availability of phosphate the line Pa36 might show a large response to colonization by AMF.

To investigate their phosphate dose-response relationship, we grew the maize lines $\mathrm{Pa} 36$ and $\mathrm{Oh} 43$ with or without AMF inoculation at several levels of phosphate availability as described in "Materials and methods". We found that the level of mycorrhizal colonization changed with phosphate availability, but there was no significant difference between the two lines in the average total colonization (not shown). We fitted the performance of non-colonized and mycorrhizal plants with sigmoid curves as described by Janos (2007) according to the equation:

$$
\begin{aligned}
\text { Weight } & =\frac{m}{1+\left(B \times e^{C \times \text { Phosphate })}\right.}, \text { where } B=\frac{m-n}{n} \text { and } \\
C & =\ln \left(\frac{n}{m-n}\right) \times \frac{1}{f}
\end{aligned}
$$

where $m$ is the maximum level of performance (the asymptote), $n$ is the performance with no additional phosphate (the $y$-axis intercept), and $f$ is the phosphate level at the point of inflection (Fig. 4, Supplemental methods). We employed this form of the logistic equation to incorporate parameters having simple biological interpretations.

To estimate dependence (Janos 2007) of the lines Oh43 and Pa36, Eq. 12 was re-arranged to calculate the level of phosphate required to sustain an arbitrary, low percentage

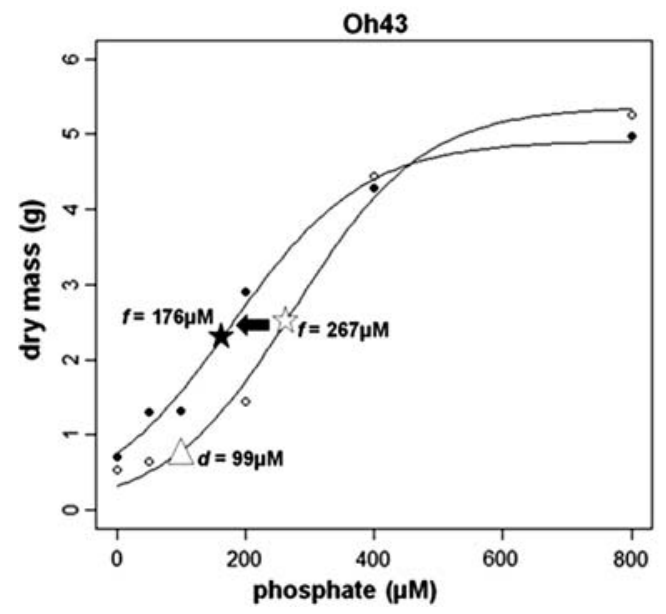

Fig. 4 Performance (plant dry-mass) curves for maize plants of the lines Oh43 and Pa36 grown in the presence (filled circles) or absence (open circles) of Glomus mosseae, across a range of phosphate availabilities. Plants were grown as described in "Materials and methods". Dose-response curves were fitted using a logistic equation of the maximum performance of non-colonized plants. To sustain $15 \%$ performance, the values were $99 \mu \mathrm{M}$ for Oh43 and $26 \mu \mathrm{M}$ for Pa36 (Fig. 4), indicating that Pa36 indeed is less dependent than $\mathrm{Oh} 43$, possibly as a result of higher grain weight (1,000-grain weight: Oh43, $234 \mathrm{~g}$; Pa36, $429 \mathrm{~g}$ ). Even if the dependence difference were entirely attributable to grain weight, however, that does not preclude there also being a mycorrhiza-specific component to the variation observed between the lines. The benefits of mycorrhiza colonization, considered across a range of phosphate levels, may be manifested as a left shift of the dose-response curve of mycorrhizal plants compared to that of non-colonized plants, reflecting mycorrhizal plants given less phosphate than to non-colonized plants performing equally to them (an "horizontal" comparison) (Janos 2007). The magnitude of such a shift can be quantified by comparison of the inflection point estimates for the $M$ and NC curves (Fig. 4). The line Oh43 shows a clear shift, corresponding to improved performance of mycorrhizal plants throughout the near-linear rise phase of the curve (Oh43: $\mathrm{NC}, \quad f=267 \mu \mathrm{M}, \quad \mathrm{SE}=20.6 ; \quad M$, $f=176 \mu \mathrm{M}, \mathrm{SE}=14.7)$. In contrast, there is little shift for the line Pa36, and no significant difference in the position of the inflection points estimated for $M$ and NC plants (Pa36: NC, $f=237 \mu \mathrm{M}, \mathrm{SE}=23.3 ; M, f=271 \mu \mathrm{M}$, $\mathrm{SE}=41.0$ ). Thus, there is no evidence for a strong mycorrhiza effect at any point along the Pa36 doseresponse curve, providing support for its selection as a low-responding parent. Consequently, we have begun to generate populations derived from $\mathrm{Oh} 43$ and $\mathrm{Pa} 36$ to further investigate the genetic basis of the phenotypic differences observed in mycorrhiza effect.

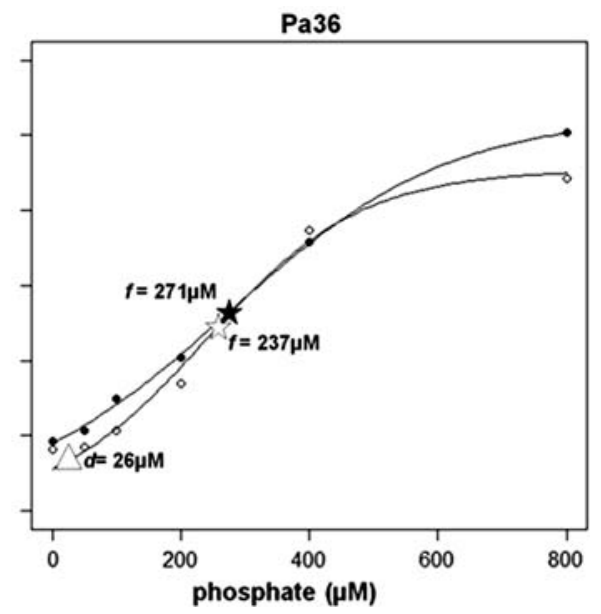

as described in the text. The phosphate requirement for $15 \%$ performance of non-colonized plants $(d$, open triangle $)$ and the positions of the inflection points $(f)$ for both colonized (filled star) and non-colonized (open star) curves are marked. The left shift observed for Oh43 is marked with a filled arrow 


\section{Discussion}

We have presented a method for partitioning genetic variance linked to differences in the performance of mycorrhizal plants into that which is correlated with variation in non-colonized performance and residual, specific variation which we propose to be of greatest interest to plant breeders. Stated informally, we seek plant lines that show a level of performance when mycorrhizal which is remarkable given their level of performance when non-colonized. Although primarily descriptive and best suited to a simple ranking of lines, our method could be adapted to consider error variation and thereby permit statistical estimation of variance components.

It is informative to compare our method with the oftenused ranking of lines on the basis of relative performance increase in the presence of mycorrhiza $\left(R^{\prime}\right)$. We have demonstrated the potential for a linear model to describe the relationship between $M$ and NC, but have shown that the relative measure $R^{\prime}$ may proffer misleading abstractions of the relationship. Varieties that conform to a population trend well described by regression of $M$ against NC can appear to be outliers on the basis of ranking by $R^{\prime}$. In the datasets we have examined, this effect is most pronounced for extreme values of $\mathrm{NC}$, corresponding to a poor linear fit to the power relationship between $R^{\prime}$ and $\mathrm{NC}$ at the extremes of the curve. We suggest that this potential inaccuracy, especially the systematic over-estimation of mycorrhiza benefit in highly dependent varieties, has complicated the interpretation of the published data sets we have re-analyzed.

Variation in the performance of crop varieties grown under low-phosphate conditions has been well documented (e.g., Da Silva and Gabelman 1992; Koide et al. 1988; Zhu et al. 2001), and such variation greatly affects measures such as $R$ and $R^{\prime}$. The analyses by Kaeppler et al. (2000), Hetrick et al. (1995), and others (e.g., Koide et al. 1988; Zhu et al. 2001) have recognized that tolerance of soil infertility reduces the benefit of mycorrhizal colonization. Although we have suggested that the use of $R^{\prime}$ exaggerates the importance of dependence differences in assessing variation in mycorrhiza effect, we do not contest that a highly dependent individual acquires a greater benefit from mycorrhizal colonization than a less-dependent one, nor do we question the physiological and ecological significance of such a responsiveness measure. Notwithstanding, plant breeding seeks to identify and select, not at the level of the whole plant but at the level of underlying genetic variation, and for that purpose interpretation of responsiveness measures can be problematic. In particular, we caution against assuming that little-responsive varieties lack "responsiveness genes" or that selective forces necessarily have acted against such a variety's ability to form functional mycorrhizas. Our interpretation is consistent with physiological studies that demonstrate mycorrhiza functionality in certain crop varieties even in the absence of growth effects (Smith et al. 2003).

With our dose-response data for maize lines Oh43 and Pa36, we have touched on the importance of measures of $M$ and NC for meaningful comparisons. Although informative results can be obtained from data taken within a single environment, a range of environments may be required to understand truly the nature of variation in the trait of interest (Schlichting and Pigliucci 1998). The simplest approach to analysis of full dose-response relationships may be to estimate a small number of summary statistics from fitted curves, although for a mycorrhiza effect, finding the differences between dose-response relationships with and without mycorrhizas complicates the analysis of variation. Nevertheless, a summary approach can be applied, for example, by using the positions of the inflection points of the $M$ and NC curves, or by using $R_{\max }$ as suggested by Janos (2007). An alternative to the use of a single summary statistic is to analyze variation in the dose-response curves as a whole. For example, the technique of template mode variation (TMV) analysis quantifies the deviation of each curve from a template curve typical of the population (Izem and Kingsolver 2005). One advantage of the TMV approach is that variation is broken down into a number of modes with no prior assumption as to which is the most important. Covariance of mode variation between mycorrhizal and non-mycorrhizal plants could be analyzed as we have proposed.

Our proposal to partition variation in mycorrhiza effect is motivated by advances in mycorrhiza biology that suggest there are plant molecular components that specifically characterize non-colonized or colonized plants. For example, physiological studies have revealed two distinct pathways of phosphate acquisition: a direct phosphate uptake pathway that functions in the absence of colonization, and a second mycorrhizal pathway that is activated following the establishment of symbiosis (Smith et al. 2003). The latter pathway can predominate in colonized plants and may completely suppress the direct pathway (Li et al. 2006; Smith et al. 2003, 2004, 2009). In consequence, variation in direct phosphate uptake need not affect phosphate uptake by colonized plants. Furthermore, certain transporters function uniquely, or predominantly, in mycorrhizal plants, while others have been linked with the direct pathway (Bucher 2007; Javot et al. 2007). Isoforms that accumulate specifically in mycorrhizal plants have been identified among the members of other protein families including aquaporins (Uehlein et al. 2007), membrane localized $\mathrm{H}^{+}$-ATPases (Gianinazzi-Pearson et al. 2000; Krajinski et al. 2002), chitinases (Salzer et al. 2000), lectins (Frenzel et al. 2005), and germin-like proteins (Doll et al. 
2003). Molecular analyses collectively suggest genetic potential for independent variation in the performance of colonized versus non-colonized plants. Allelic variation in such genes would provide a molecular basis for the specific variation we identify with our regression analysis.

The increasing availability of molecular resources for major crop species is driving new approaches to the understanding of crop development and the selection of future varieties; sequence data facilitate the generation of markers and the identification of candidate genes; expression platforms are taking the measurement of traits from the purely morphological to the level of gene action; quantitative trait loci (QTL) and association analyses are beginning to make the link between traits and the genes that govern them. The underlying aim, however, remains that of correlating phenotypic and genetic information. Accurate assessment of the trait of interest thereby remains the foundation of any successful study. We hope that our suggestions for partitioning mycorrhiza effect will aid in the clear identification of meaningful variation, and thereby will support modern molecular breeding approaches in improving the utility of AMF in agriculture.

Acknowledgments We thank Tom Juenger (University of Texas), Jerome Goudet (University of Lausanne), and anonymous reviewers for helpful comments during preparation of this manuscript, Shawn Kaeppler (University of Wisconsin) for providing maize seed, and Blaise Tissot (University of Lausanne) for help with plant growth. R.S. thanks César Sawers for additional assistance. This work was supported by Swiss National Science Foundation grant PP00A-110874 to R.S. and U.P. and a European COST action 870 grant to M. G.

\section{References}

Bucher M (2007) Functional biology of plant phosphate uptake at root and mycorrhiza interfaces. New Phytol 173:11-26

Da Silva AE, Gabelman WH (1992) Screening maize inbred lines for tolerance of low-P stress conditions. Plant Soil 146:181-187

Delgado A, Scalenghe R (2008) Aspects of phosphorus transfer from soils in Europe. J Plant Nutr Soil Sci (Zeitschrift Fur Pflanzenernahrung Und Bodenkunde) 171:552-575

Doll J, Hause B, Demchenko K, Pawlowski K, Krajinski F (2003) A member of the germin-like protein family is a highly conserved mycorrhiza-specific induced gene. Plant Cell Physiol 44:1208-1214

Evenson RE, Gollin D (2003) Assessing the impact of the green revolution, 1960 to 2000 . Science 300:758-762

Falconer DS (1990) Selection in different environments-effects on environmental sensitivity (reaction norm) and on mean performance. Genet Res 56:57-70

Frenzel A, Manthey K, Perlick AM, Meyer F, Puhler A, Kuster H, Krajinski F (2005) Combined transcriptome profiling reveals a novel family of arbuscular mycorrhizal-specific Medicago truncatula lectin genes. Mol Plant Microbe Interact 18:771-782

Gianinazzi-Pearson V, Arnould C, Oufattole M, Arango M, Gianinazzi S (2000) Differential activation of H+-ATPase genes by an arbuscular mycorrhizal fungus in root cells of transgenic tobacco. Planta 211:609-613

Grace EJ, Cotsaftis O, Tester M, Smith FA, Smith SE (2009) Arbuscular mycorrhizal inhibition of growth in barley cannot be attributed to extent of colonization, fungal phosphorus uptake or effects on expression of plant phosphate transporter genes. New Phytol 181:938-949

Güimil S, Chang H, Zhu T, Sesma A, Osbourn A, Roux C, Ioannidis V, Oakely EJ, Docquier M, Descombes P, Briggs SP, Paszkowski U (2005) Comparative transcriptomics of rice reveals an ancient pattern of response to microbial colonization. Proc Natl Acad Sci 102:8066-8070

Gutjahr C, Banba M, Croset V, An K, Miyao A, An G, Hirochika H, Imaizumi-Anraku H, Paszkowski U (2008) Arbuscular mycorrhiza-specific signaling in rice transcends the common symbiosis signaling pathway. Plant Cell 20:2989-3005

Hetrick BAD, Wilson GWT, Cox TS (1992) Mycorrhizal dependence of modern wheat varieties, landraces, and ancestors. Can J Bot 70:2032-2040

Hetrick BAD, Wilson GWT, Gill BS, Cox TS (1995) Chromosomal location of mycorrhizal responsive genes in wheat. Can J Bot 73:891-897

Hetrick BAD, Wilson GWT, Cox TS (1996) Mycorrhizal response in wheat cultivars: relationship to phosphorous. Can J Bot 74:19-25

Hoagland D, Broyer T (1936) General nature of the process of salt accumulation by roots with description of experimental methods. Plant Physiol 11:451-507

Izem R, Kingsolver JG (2005) Variation in continuous reaction norms: quantifying directions of biological interest. Am Nat 166:277-289

Janos DP (2007) Plant responsiveness to mycorrhizas differs from dependence upon mycorrhizas. Mycorrhiza 17:75-91

Javot H, Pumplin N, Harrison MJ (2007) Phosphate in the arbuscular mycorrhizal symbiosis: transport properties and regulatory roles. Plant Cell Environ 30:310-322

Kaeppler SM, Parke JL, Mueller SM, Senior L, Stuber C, Tracy WF (2000) Variation among maize inbred lines and detection of quantitative trait loci for growth at low phosphorous and responsiveness to arbuscular mycorrhizal fungi. Crop Sci 40:358-364

Khasawneh FE, Sample EC, Kamprath EJ (eds) (1986) The role of phosphorus in agriculture. American Society of Agronomy, Madison, USA

Koide RT, Li M, Lewis J, Irby C (1988) Role of mycorrhizal infection in the growth and reproduction of wild vs. cultivated plants. I. Wild vs. cultivated oats. Oecologia 77:537-543

Krajinski F, Hause B, Gianinazzi-Pearson V, Franken P (2002) Mthal, a plasma membrane H+-ATPase gene from Medicago truncatula shows arbuscule-specific induced expression in mycorrhizal tissue. Plant Mol Biol 4:754-761

Li HY, Smith SE, Holloway RE, Zhu YG, Smith FA (2006) Arbuscular mycorrhizal fungi contribute to phosphorus uptake by wheat grown in a phosphorus-fixing soil even in the absence of positive growth responses. New Phytol 172:536-543

Li HY, Smith SE, Ophel-Keller K, Holloway RE, Smith FA (2008) Naturally occurring arbuscular mycorrhizal fungi can replace direct $\mathrm{P}$ uptake by wheat when roots cannot access added $\mathrm{P}$ fertiliser. Funct Plant Biol 35:124-130

Lynch M, Walsh B (1998) Genetics and analysis of quantitative traits. Sinauer, Sunderland, MA

Paszkowski U, Boller T (2002) The growth defect of lrt1, a maize mutant lacking lateral roots, can be complemented by symbiotic fungi or high phosphate nutrition. Planta 214:584-590

Salzer P, Bonanomi A, Beyer K, Vogeli-Lange R, Aescherbacher RA, Lange J, Wiemken A, Kim D, Cook DR, Boller T (2000) Differential expression of eight chitinase genes in Medicago 
truncatula roots during mycorrhiza formation, nodulation and pathogen infection. MPMI 13:763-777

Sawers RJ (2009) Breeding for reaction. In: Huttunen N, Sinisalo T (eds) Plant Breeding. Nova Publishers, New York

Sawers RJ, Gutjahr C, Paszkowski U (2008) Cereal mycorrhiza: an ancient symbiosis in modern agriculture. Trends Plant Sci 13:93-97

Schlichting CD, Pigliucci M (1998) Phenotypic evolution: a reaction norm perspective. Sinauer, Sunderland, MA

Smith SE, Read DJ (2007) Mycorrhizal symbiosis, 3rd edn. Academic Press, London

Smith SE, Smith FA, Jakobsen I (2003) Mycorrhizal fungi can dominate phosphate supply to plants irrespective of growth responses. Plant Phys 133:16-20

Smith S, Smith F, Jacobsen I (2004) Functional diversity in arbuscular mycorrhizal (AM) symbioses: the contribution of the mycorrhizal $\mathrm{P}$ uptake pathway is not correlated with mycorrhizal responses in growth or total P uptake. New Phytol 162:511-524
Smith FA, Grace EJ, Smith SE (2009) More than a carbon economy: nutrient trade and ecological sustainability in facultative arbuscular mycorrhizal symbioses. New Phytol 182:347-358

Uehlein N, Fileschi K, Eckert M, Bienert GP, Bertl A, Kaldenhoff R (2007) Arbuscular mycorrhizal symbiosis and plant aquaporin expression. Phytochemistry 68:122-129

Wright DP, Scholes JD, Read DJ, Rolfe SA (2005) European and African maize cultivars differ in their physiological and molecular responses to mycorrhizal infection. New Phytol 167:881-896

Zhu Y, Smith S, Barritt A, Smith F (2001) Phosphorus (P) efficiencies and mycorrhizal responsiveness of old and modern wheat cultivars. Plant Soil 237:249-255

Zhu Y-G, Smith FA, Smith SE (2003) Phosphorous efficiency and responses of barley (Hordeum vulgare L.) to arbuscular mycorrhizal fungi grown in highly calcareous soil. Mycorrhiza 13:93-100 